\title{
77 GHz Screen Printed, Flexible, Beam-Switching Antenna Array for Wearable Radar Applications
}

\author{
Azat Meredov, Kirill Klionovski, and Atif Shamim \\ Division of Computer, Electrical, and Mathematical Sciences and Engineering \\ King Abdullah University of Science and Technology (KAUST) \\ Thuwal 23955-6900, Saudi Arabia \\ azat.meredov@kaust.edu.sa,kirill.klionovski@kaust.edu.sa, atif.shamim@kaust.edu.sa
}

\begin{abstract}
Wave radars are widely used in the automotive industry, but the concept is rather new for wearable applications, such as detection of obstacles for visually impaired people. Beamswitching techniques for radar antennas, typically, are either complicated or bulky, whereas for wearable applications, simpler methods are desirable. In this paper, we present a high gain antenna array with a low profile and a simple beam-switching design. The concepts of parasitic antenna array and partially reflective surface have been combined to simultaneously achieve a narrow beam (higher gain) as well as beam-switching. Additive manufacturing (screen printing) has been utilized which ensures flexibility (essential for wearable applications) and a lower cost. A $\pm 30^{\circ}$ beam switching and 11-13 dB realized gain has been achieved, which is deemed suitable for the given application.
\end{abstract}

Keywords-Parasitic antenna array, partially reflective surface, mm-wave antenna.

\section{INTRODUCTION}

Radars are used for a wide range of detection and navigation applications, such as detecting airplane, ships, space crafts, and navigating motorized vehicles, robotics, etc. [1]. The antenna used for the radar defines the scan range of the radar. A simple antenna can give detection in one direction, but to have detection in multiple directions antenna beam should be steerable, which can be achieved by mechanical or electrical steering $[1,2]$. The mechanically steered antennas are bulky and require angle tracking to find the location. On the other hand, electrically steerable antennas can be low profile and less bulky.

Electrical beam steering can be performed using methods such as frequency scanning, the use of phase shifters, Butler or Blass matrices, Rotman lens, or beam switching [3]. Beam scanning using phase shifters at mm-wave frequencies is expensive, narrow band, and has high losses. The Butler matrix requires to design couplers, crossovers, and delay lines. This large feed network adds additional losses to the system. Rotman lens typically has a large size.

The parasitic antenna array concept, on the other hand, can be used to realize a small as well as a simple beam-switching antenna system without adding losses due to a large feeding network. The parasitic antenna array comprises a single active (or driven) element and some parasitic elements to enable beam-switching. When parasitic antennas are placed close enough to the driven antenna, the current is induced in them due to the parasitic coupling, thus changing the overall radiation pattern of the antenna array.
In literature, the parasitic antenna concept has been used to switch the antenna beam for different applications in [4-7]. The major drawback of these works is the wide beam width of the main lobe. Even though the wider beam width was not an issue for their particular applications, a radar antenna needs a narrower beam to distinguish the objects from different directions. Adding a partially reflective surface (PRS) on top of the designed antenna is expected to resolve this problem. A PRD can help to increase the antenna gain and decrease the main lobe beam width of the antenna. In this work, we have incorporated a PRS structure to the parasitic antenna array design. By utilizing the parasitic antennas and the PRS, we have achieved a simple beam-switching antenna (as a proof of concept) that can operate in three directions $\left(-30^{\circ}, 0^{\circ}, 30^{\circ}\right)$ with a narrow beam width. Screen printing on a flexible substrate has been utilized to achieve the required flexibility for wearable applications.

\section{BEAM SWITCHING ANTENNA DESIGN}

For the radar antenna, the intention is to design a simple antenna which can detect objects in three directions $(-30,0$, and 30 degrees). Antennas have been designed on a flexible PreFlex substrate with a dielectric constant of 2.6 and a loss tangent of 0.0044 . For this purpose, we have used three antennas with beams in the desired three directions. The bore sight radiation has been obtained from a square patch antenna. To achieve right and left directed beams we have utilized parasitic antenna array concept, where shorted and open patches are used to direct the beam. In a parasitic antenna array, current is induced in the passive elements by placing them very close to the driven antenna. The shorted parasitic patches help to direct the beam in the same direction where they are located and the open parasitic patches help to direct the beam in a direction opposite to their location. Thus, to direct the beam of the antenna we have used four shorted patches in one side of the active antenna and four open patches on the other side to have the beam directed around $30^{\circ}$. The parasitic antenna array has helped us to avoid the distribution losses present in the standard antenna arrays.

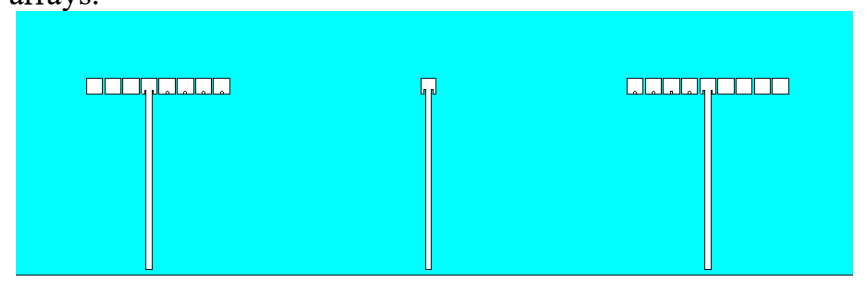

Fig. 1: Proposed antenna to obtain three beams. 
The designed patch antenna has a realized gain of $7 \mathrm{dBi}$ and a half power beam width (HPBW) of $68^{\circ}$, and the parasitic antenna array has a realized gain of $6.6 \mathrm{~dB}$ and HPBW of $97^{\circ}$. The beam in the other direction $\left(-30^{\circ}\right)$ has been obtained by switching the shorted parasitic elements with the open parasitic elements. Proposed three antennas are shown in Figure 1. With this, we have achieved three beams, but the antenna beam widths are too wide to distinguish the objects in different directions.

To reduce the beam widths of the antennas, we have used a PRS structure on top of the antennas. In PRS multiple reflections are happening between the ground plane and the PRS, and when the phase of the transmitted signals are same, they will add up and increase the antenna gain. We used periodic square patches to form the PRS layer. The PRS is placed on top of $\lambda / 2 \approx 1.3 \mathrm{~mm}$ thick superstrate to maintain a low profile as well as flexibility of the antennas. The PRS has a dimension of $750 \mu \mathrm{m}$ for the single patch antenna and has 850 $\mu \mathrm{m}$ size for the parasitic antenna arrays. This way the antenna beam width has been decreased significantly. The gain of parasitic antenna array has increased from 6.5 to $11 \mathrm{dBi}$, while the single patch gain has increased from 7 to $13 \mathrm{dBi}$. The designed parasitic antenna with PRS has $-8 \mathrm{~dB}$ side lobe level. To decrease the side lobe level of the parasitic antenna array with PRS, we have replaced one half of the PRS with a metal sheet, which decreased the side lobe level from $-8 \mathrm{~dB}$ to $-11 \mathrm{~dB}$. The H-plane radiation pattern of the three antennas are shown in Fig. 2 and the final antenna designs with the PRS are shown in Fig. 3(a).

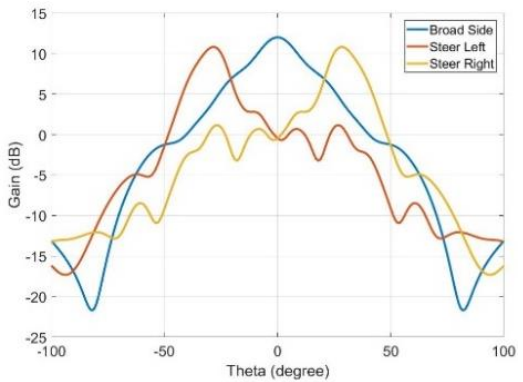

Fig. 1. Proposed parasitic antenna array, and the H-plane radiation pattern of the single patch and parasitic antenna array with FSS.

\section{SCREen PRINTEd PROTOTYPe AND MEASURED RESUltS}

The antenna has been fabricated using screen printing. To align the multilayer antenna and to create shorting vias, the substrate has been processed through laser. With the help of laser we have formed holes for via and alignment marks for the alignment of layers on the antenna substrate. The vias have been filled with DuPont PE 873 silver ink. Then the antenna, ground and PRS layers have been printed separately with the silver ink using the alignment cameras and alignment marks. After printing, layers have been cured at $120^{\circ} \mathrm{C}$ in the oven for 10 minutes. Finally, all the antenna layers are stacked together to realize the final antenna.

A microstrip to CPW transition has been added to the antennas to enable testing of the antenna through RF probes. The fabricated antenna has been tested in a special anechoic chamber designed for probe fed antennas. Fabricated antennas with PRS are shown in Fig. 2(a) and the measured radiation patterns are shown in Fig. 2(b,c). The results obtained from measurements are well aligned with the predicted simulation results.

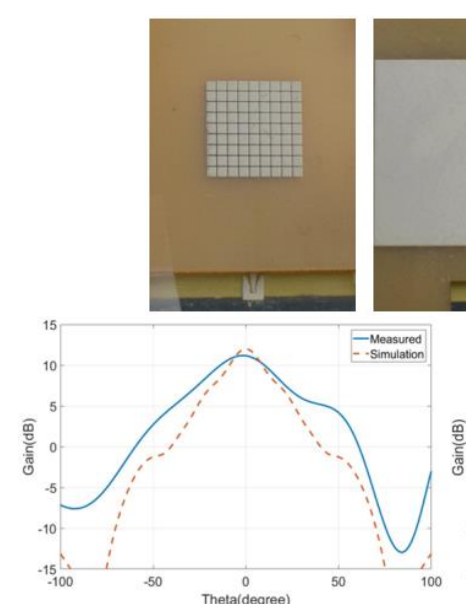

(b)

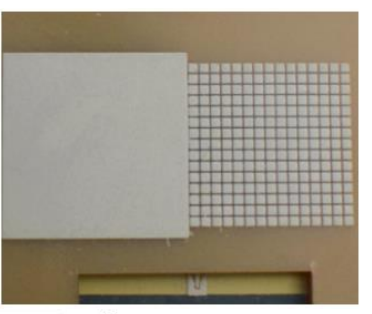

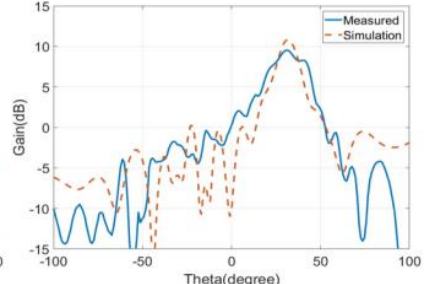

(c)
Fig. 2. (a) Fabricated antenna samples, (b) Radiation pattern of the measured single patch antenna with PRS, and (c) Radiation pattern of the parasitic antenna array with PRS.

\section{CONCLUSION}

In this work, a low profile, flexible, and the high gain antenna has been designed. $\mathrm{A} \pm 30^{\circ}$ beam switching has been obtained. The antenna has a realized gain of $11-13 \mathrm{dBi}$ in the given angle range. Antennas have been realized by screen printing. A microstrip line to coplanar waveguide (CPWG) transmission has been added to test the antennas by probing. The antenna radiation patterns have been tested and are in good agreement with the simulations.

\section{ACKNOWLEDGMENT}

The authors would like to thank the PremixGroup team for their extensive support on the material information.

\section{REFERENCES}

[1] Skolnik, M.I., Radar handbook. 1970

[2] Fennigkoh, L., Wiley Encyclopedia of Electrical and Electronics Engineering. Biomedical Instrumentation \& Technology, 2002. 36(5): p. 355-355.

[3] Ku, B.-H., et al., A 77-81-GHz 16-Element Phased-Array Receiver With $\$ 1$ pm $\{\mid \text { hbox }\{50\}\}^{\wedge}\{\mid c i r c\}$ \$ Beam Scanning for Advanced Automotive Radars. IEEE Transactions on Microwave Theory and Techniques, 2014. 62(11): p. 2823-2832.

[4] Preston, S.L., et al., Base-station tracking in mobile communications using a switched parasitic antenna array. IEEE Transactions on Antennas and Propagation, 1998. 46(6): p. 841-844.

[5] Preston, S., et al., Electronic beam steering using switched parasitic patch elements. Electronics Letters, 1997. 33(1): p. 7-8.

[6] Jusoh, M., et al., Reconfigurable four-parasitic-elements patch antenna for high-gain beam switching application. IEEE Antennas and Wireless Propagation Letters, 2014. 13: p. 79-82.

[7] Sabapathy, T., et al., A ground-plane-truncated, broadly steerable YagiUda patch array antenna. IEEE Antennas and Wireless Propagation Letters, 2016. 15: p. 1069-1072 